\title{
Campanienne (céramique)
}

J.-P. Morel

\section{OpenEdition}

Journals

Édition électronique

URL : http://journals.openedition.org/encyclopedieberbere/2044

DOI : 10.4000/encyclopedieberbere.2044

ISSN : 2262-7197

\section{Éditeur}

Peeters Publishers

\section{Édition imprimée}

Date de publication : 1 septembre 1992

Pagination : 1720-1725

ISBN : 2-85744-581-4

ISSN : 1015-7344

\section{Référence électronique}

J.-P. Morel, « Campanienne (céramique) », Encyclopédie berbère [En ligne], 11 | 1992, document C12, mis en ligne le 01 avril 2013, consulté le 25 septembre 2020. URL : http://journals.openedition.org/ encyclopedieberbere/2044; DOI : https://doi.org/10.4000/encyclopedieberbere.2044

Ce document a été généré automatiquement le 25 septembre 2020

(c) Tous droits réservés 


\title{
Campanienne (céramique)
}

\author{
J.-P. Morel
}

1 Le terme de "céramique campanienne" désigne communément l'ensemble des céramiques à vernis noir de la Méditerranée occidentale à l'époque hellénistique, indépendamment de leur origine réelle. La situation de ces céramiques en Afrique quant à la production et au commerce, longtemps brouillée par des ignorances, des erreurs et des préjugés, commence à apparaître avec une certaine netteté.

Une opinion répandue veut par exemple que la bataille d'Himère, au cours de laquelle les troupes grecques défirent les Carthaginois en Sicile en 480, ait entraîné pour l'Afrique une longue période de fermeture aux produits helléniques. Mais en fait, la céramique attique à vernis noir arrive dans le Maghreb au cours du ve siècle en quantités notables. Ces importations augmentent et culminent au IV siècle. Beaucoup des vases attiques à vernis noir de cette époque trouvés à Carthage portent des chiffres grecs gravés, et quelques-uns combinent ces chiffres avec des lettres puniques, juxtaposition que l'on a trouvée aussi sur un vase de l'épave punique de El Sec, à Palma de Majorque. De tels documents suggèrent l'existence de circuits gréco-puniques, ou peut-être même de firmes mixtes, pour la diffusion de ces céramiques vers l'Occident ; ils confirment le rôle joué par les «Phéniciens" dans le commerce de la céramique attique, mentionné par un texte du Pseudo-Scylax (112) qui concerne plus particulièrement la côte atlantique de l'Afrique.

3 La céramique attique à vernis noir continue à être importée au $\mathrm{III}^{\mathrm{e}}$ siècle (et même,

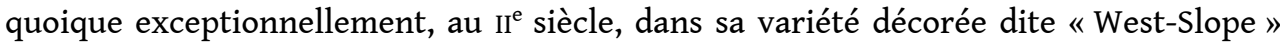
ou «du Versant occidental»). Aux IV et ${ }^{\mathrm{e}}{ }^{\mathrm{e}}$ siècles, elle suscite dans l'ensemble de l'Afrique du Nord des imitations locales. Ces dernières combinent des formes empruntées au répertoire athénien avec des caractéristiques techniques clairement puniques (fréquence des pâtes grises, des surfaces rougeâtres ou grisâtres, des « zones d'empilement » provoquées par l'entassement des vases dans le four). Les abords de Carthage et la Tripolitaine sont les deux régions où ces imitations sont le mieux attestées, et une fabrique en a été trouvée à la Rabta à Tunis. Mais c'est aussi de la céramique attique que s'inspire dans une large mesure au $\mathrm{III}^{\mathrm{e}}$ siècle un atelier découvert 
à Kouass, dans la région d'Asilah, au Maroc. Les produits de ces officines connaissent une diffusion strictement régionale, voire locale.

4 A l'extrême fin $\mathrm{du} \mathrm{IV}^{\mathrm{e}}$ siècle, entre en jeu un nouveau protagoniste du commerce méditerranéen : Rome. Sa présence sur les marchés africains est révélée par les vases de l'atelier des petites estampilles. Ces vases romains ou en tout cas latiaux sont exportés dans l'aire punicisante, aussi bien à Carthage même ou sur les sites voisins (jusqu'à Utique et Hadrumète) qu'en Tripolitaine, en Sicile occidentale, en Sardaigne, enfin en Espagne sud-orientale. Carthage recèle même quelques exemplaires des rares vases à décor surpeint de cet atelier, connus en Italie sous le nom de pocola. Très peu d'autres céramiques italiennes parviennent alors en Afrique. C'est encore à Carthage que l'on note autour du début du III $^{\mathrm{e}}$ siècle, en quantités infimes, de la céramique dite «de Gnathia » (tarentine en réalité), et quelques vases siciliotes. Plus tard, dans la seconde moitié $\mathrm{du} \mathrm{III}^{\mathrm{e}}$ siècle, arriveront dans cette ville de rares exemplaires de la céramique de Calés décorée de reliefs (gutti, patères à ombilic), ainsi qu'une petite patère à frise de masques en relief provenant vraisemblablement de Teano, en Campanie septentrionale.

5 Beaucoup plus important est le processus par lequel, toujours au cours du $\mathrm{III}^{\mathrm{e}}$ siècle, apparaissent et se développent en certaines régions du monde punique, de nouvelles céramiques locales à vernis noir, parfois de grande qualité. Deux zones sont particulièrement concernées par ce phénomène: la Sicile occidentale, autour de Lilybée, Palerme et Solunto, et l'Afrique, notamment à Carthage.

Plat à poisson de Gastel. Photo M. Bovis.

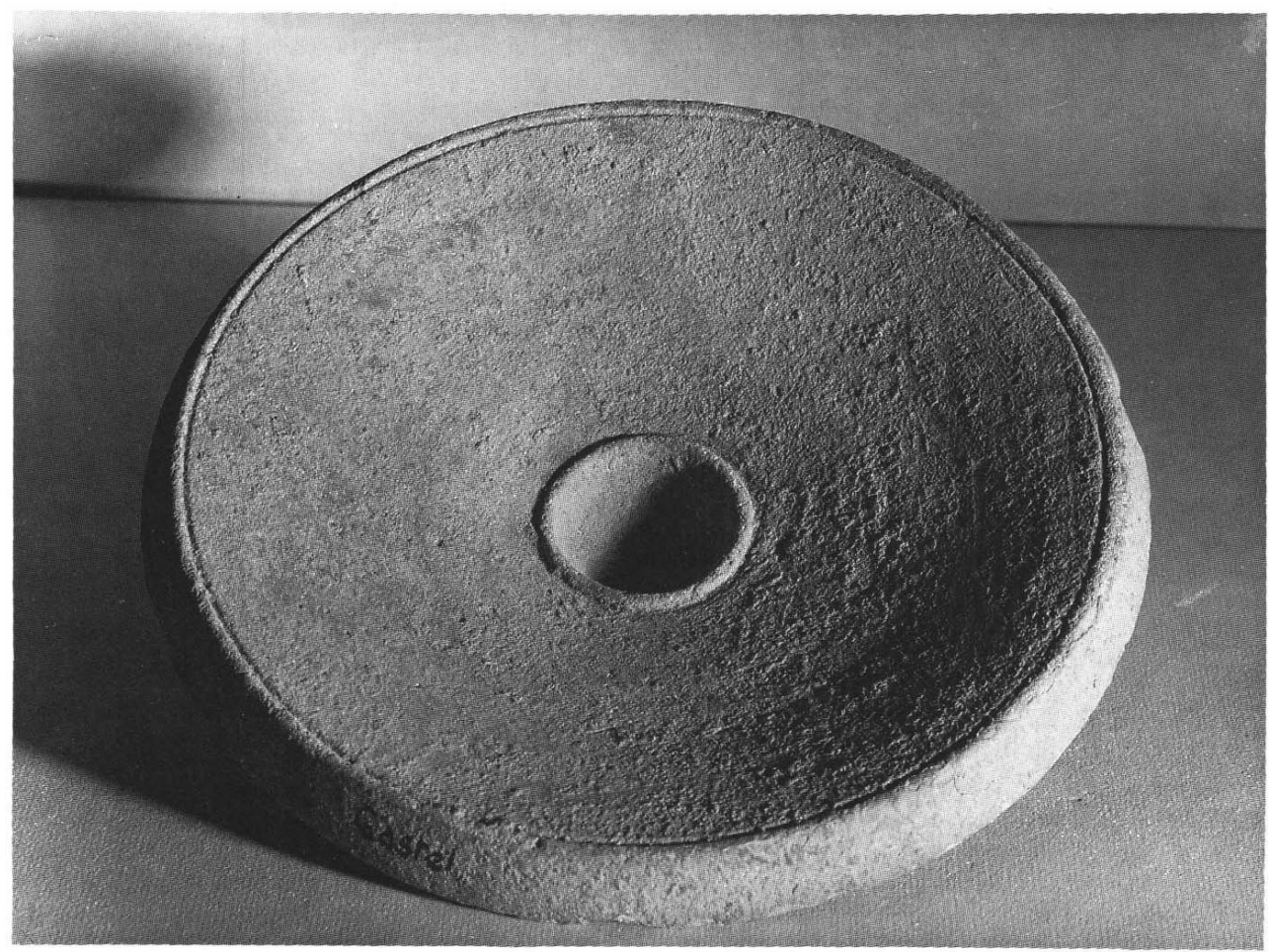

6 Parmi les productions à vernis noir de cette dernière ville, qui rompent avec les modèles attiques pour assumer une réelle originalité, il faut mentionner particulièrement le groupe "Byrsa 401 ", caractérisé par une pâte jaune très pâle proche de celle des produits corinthiens archaïques, et par un vernis fragile qui 
s'écaille au point de disparaître presque complètement; et le groupe "Byrsa 661 ", aux parois minces, au beau vernis solide d'un noir profond, aux formes originales. L'Afrique acquiert alors une certaine autonomie et une véritable maîtrise dans le domaine de la céramique à vernis noir, mais les quantités produites sont plutôt faibles, pour les productions précitées comme pour celles qui leur correspondent dans d'autres régions d'Afrique. Parmi les formes les mieux représentées, mentionnons des coupes à anses de type hellénistique, à décor surpeint sous le bord interne; des bols aux profils variés, dont certains reposent sur trois supports en forme de coquilles ou de masques; de petites cruches à panse godronnée très renflée vers le bas ou vers le haut, à col très étroit, à bouche trilobée; des plats à poisson. Parmi les décors - souvent des palmettes ou des rosettes de types divers -, apparaissent fréquemment des timbres cruciformes simulant quatre palmettes groupées.

7 Une place à part revient, entre la fin du IV et le début du II siècle, à une petite série de vases plastiques à vernis noir représentant des têtes de nègres, des pieds chaussés d'une sandale, ou de nombreux autres types de personnages et d'animaux. Ils sont issus d'ateliers carthaginois, mais aussi d'autres officines, et sont présents, en petit nombre, depuis la Tunisie jusqu'au Maroc (ainsi que dans le reste de l'aire punicisante : Sicile occidentale, Sardaigne, Ibiza, littoral oriental de l'Espagne). Souvent attribués à tort à Athènes ou à Alexandrie, ils témoignent bien plutôt de l'originalité d'une production punique et punicisante de céramique à vernis noir qui a trop longtemps été négligée, quand elle n'est pas passée totalement inaperçue.

Des régions modérément imprégnées de culture carthaginoise produisent, dans l'intérieur des terres aussi bien que sur la côte, des céramiques inspirées elles aussi du répertoire méditerranéen, mais traitées avec une technique qui ne maîtrise pas encore le vernis noir ou, tout aussi bien, avec un goût qui préfère d'autres solutions chromatiques et particulièrement le rouge : citons un plat à poisson achrome de Gastel, des coupes à décor peint sur fond rouge de Lemta, des cruches de type punique, mais achromes, de ces deux sites, des vases à engobe rouge de El Hkayma. Ces céramiques « hybrides » sont particulièrement bien représentées dans le Sahel tunisien, où parfois elles empruntent plutôt leurs modèles aux formes de la céramique modelée locale.

Le passage du $\mathrm{III}^{\mathrm{e}}$ au $\mathrm{II}^{\mathrm{e}}$ siècle est marqué dans le Maghreb comme ailleurs par un bouleversement des réseaux commerciaux en ce qui concerne la céramique campanienne. Alors qu'une quasi-autarcie prévalait depuis la première guerre punique, certaines régions sont inondées après la défaite d'Hannibal par une production italienne originaire de Naples, la campanienne A. Cette dernière se caractérise par une pâte rouge-marron, par des formes empruntées essentiellement au répertoire hellénistique, par des décors rudimentaires, et fait l'objet d'une production et d'une exportation de masse. On a peine à imaginer les quantités de cette céramique qui sont parvenues en Afrique. A Carthage, où la campanienne A est arrivée plus précocement que dans beaucoup d'autres régions de la Méditerranée (vers 200 peut-être, sinon même pendant ou plutôt avant la deuxième guerre punique), une très grande proportion de la vaisselle de table de la première moitié du $\mathrm{II}^{\mathrm{e}}$ siècle provient des ateliers de Naples. La Tripolitaine, l'Algérie orientale (en particulier Hippone), et même la Cyrénaïque (Benghazi) reçoivent aussi des quantités très notables de cette campanienne A «ancienne ». Celle-ci est en revanche extrêmement rare au Maroc, région qui reste presque fermée aux importations méditerranéennes entre le IV et la fin du II siècle. 
10 On ne saurait évoquer ces apports sans souligner aussi le choix opéré par les populations africaines dans le répertoire que leur offrent les ateliers du Nord de la Méditerranée. Les Carthaginois, par exemple, privilégient, dans leurs demeures comme dans leurs tombes, les coupes véritables, pourvues d'anses (qu'il s'agisse des « boisais » de la céramique attique à vernis noir ou plus tard de coupes en campanienne $\mathrm{A}$ ), au détriment des bols sans anses. Ils fabriquent aussi des contrefaçons de ces formes, au moment même où l'Italie abandonne pour l'argent ou le bronze les coupes en terre cuite. Le vase unique et sans doute prestigieux qui constitue le mobilier d'une tombe de Bled Riat el Khémis, au Maroc, est une coupe à anses en campanienne A. Dans la vaisselle quotidienne, les plats à poisson sont également très prisés.

11 Dès le deuxième quart du II $^{\mathrm{e}}$ siècle, une autre production italienne à vernis noir vient concurrencer la campanienne $\mathrm{A}$ : la campanienne $\mathrm{B}$, une très belle céramique à pâte claire (calcaire), aux formes inspirées de techniques métalliques, originaire de l'Etrurie septentrionale maritime. Elle est présente à Carthage dès avant 146 av. n.è. Au cours de la seconde moitié du II $^{\mathrm{e}}$ siècle, la campanienne $\mathrm{B}$ sera très largement supplantée par ses imitations, voire ses contrefaçons, faites dans des ateliers campaniens comme celui de Calés. Cette campanienne «B-oïde » prédomine dans la partie occidentale du Maghreb, au Maroc ou sur un site de l'Algérie occidentale comme Tiaret, tandis qu'en Numidie la campanienne A continue à l'emporter, par exemple à Hippone : mystères des circuits commerciaux... Çà et là, on trouve aussi, en très faible quantité, la campanienne $C$, une céramique à vernis noir et pâte grise originaire de Sicile (Syracuse), la troisième des céramiques à diffusion « universelle ».

On fabrique un peu partout des imitations locales de ces campaniennes d'Italie. Très souvent, elles présentent une caractéristique frappante des productions africaines, c'est-à-dire une certaine indifférence à la couleur de leur surface, qui peut être couverte d'un vernis noir, gris, rouge, voire dépourvue de vernis, ou décorée de bandes peintes, tandis que leur pâte est aussi de couleurs variées. Elles empruntent volontiers à la campanienne B-oïde -particulièrement au Maroc - les timbres dits " en losange " ("a losanga »), figurant un carré à côtés concaves et à pointes terminées par des palmettes. Toujours au Maroc, les liens culturels et commerciaux qui, particulièrement $\mathrm{au} \mathrm{I}^{\text {er }}$ siècle, unissent cette région avec la Bétique sont évidents aussi dans le domaine des céramiques à vernis noir. 


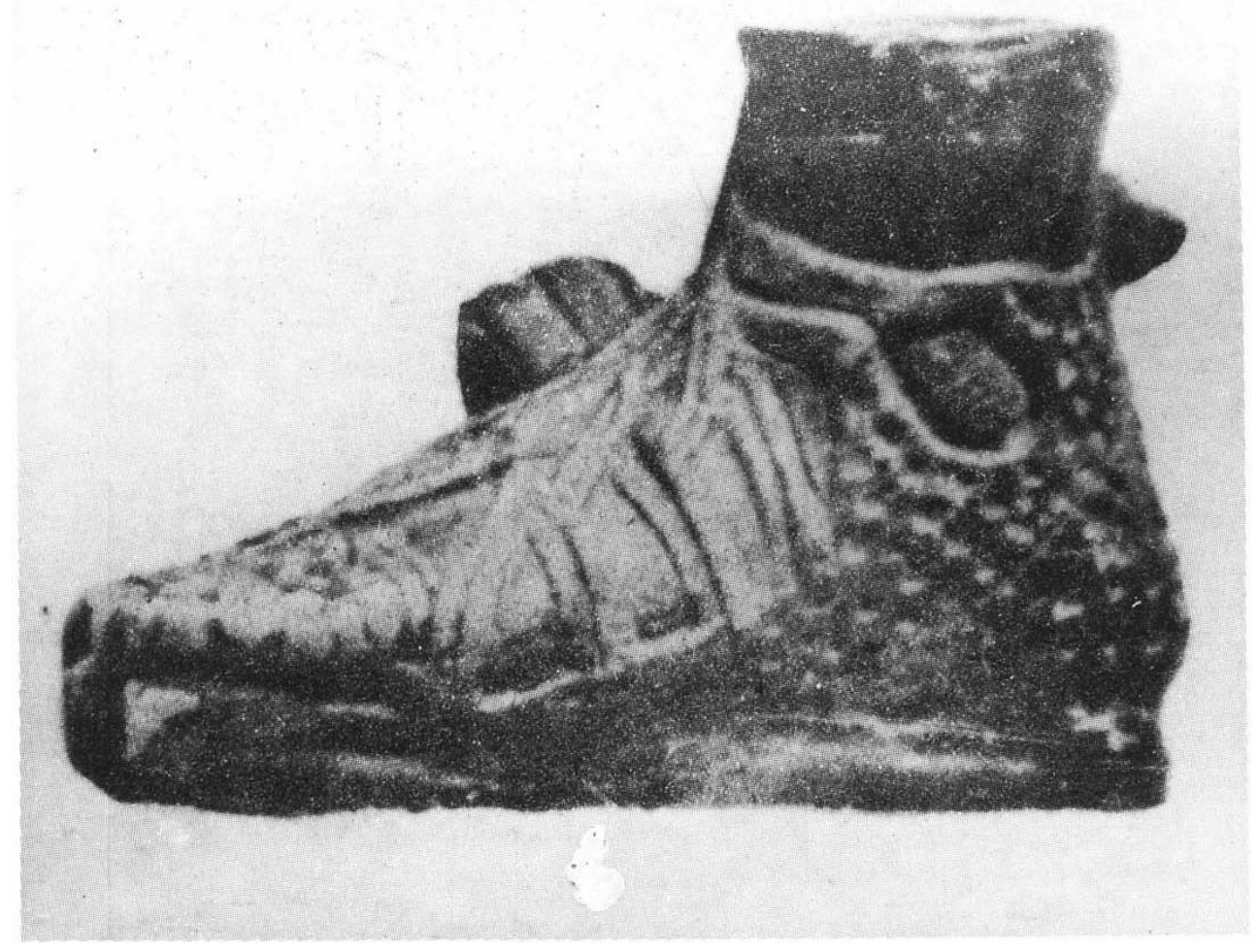

13 L'importation des campaniennes d'Italie, leur imitation dans les ateliers africains, dureront jusque vers les années $40 / 30$ av. n.è., avant que progressivement la céramique sigillée de type arétin ne les supplante. Le goût des consommateurs méditerranéens, orienté prioritairement depuis des siècles vers le vernis noir (à l'exception notable, toutefois, du monde phénico-punique amateur de vernis rouge, ce qui se traduit, on l'a $\mathrm{vu}$, dans les imitations locales), bascule alors pour très longtemps vers le rouge.

Ainsi, au long de ces quatre siècles et plus, la céramique à vernis noir du Maghreb reflète les vicissitudes du pays aussi bien que celles des modes de production et de commercialisation à travers la Méditerranée. $A u v^{e}$ et surtout $a u \mathrm{IV}^{\mathrm{e}}$ siècle, la suprématie des produits athéniens est favorisée par la participation active des marchands carthaginois à leur diffusion. Vers 300, Rome s'insère dans ce jeu pendant une trentaine d'années, avant que la première guerre punique, ici comme au Nord de la Méditerranée, ne vienne bouleverser les circuits commerciaux antérieurs et favoriser la prolifération d'ateliers locaux: les faciès régionaux sont alors très variés. Après la deuxième guerre punique, l'emprise désormais triomphante de Rome et l'irruption massive des campaniennes d'Italie oblitèrent partiellement ces particularismes céramiques. Toutefois, la persistance de petits ateliers locaux continue de donner au Maghreb une physionomie propre, elle-même diverse selon les régions. 


\section{BIBLIOGRAPHIE}

BEN YOUNES H., « La céramique "hybride" du Sahel punique », Bull, des Travaux de l'Institut National d'Archéologie et d'Art. Comptes rendus, fasc. 3, janvier-avril 1989, p. 73-86.

BOUBE J., « Introduction à l'étude de la céramique à vernis noir de Sala », Bull. d'Arch. Marocaine, XVI, 1985-1986, p. 121-190.

CADENAT P., «Établissement préromain dans la région de Tiaret (Oranie) », Ant. Afr., 6, 1972, p. 25-58.

CAMPS G., Aux origines de la Berbérie. Monuments et rites funéraires protohistoriques, Paris, 1961.

CAPUTO G., "Leptis Magna e l'industria artistica campana », Rendic. dell'Accad. di Archeol. Lett. e Belle Arti di Napoli, n.s., 35, 1960, p. 11-27.

CHELBI F., «Céramique à vernis noir de la Rabta », Latomus, 31, 1972, 2, p. 368-378 ; Céramique à vernis noir de Carthage, thèse de $3^{\mathrm{e}}$ cycle dactylographiée, Université de Provence, Aix-enProvence, 1981. « Les vases à vernis noir des nécropoles carthaginoises de la fin du ve siècle à la fin de la deuxième guerre punique ", Actes du Colloque sur la céramique antique de Carthage (Carthage, 1980), Dossier CEDAC 1, Carthage, 1982, p. 23-41.

CINTAS P., « A propos de trois vases du Musée de Sousse », Cahiers de Tunisie, 8, 1960, 3, p. 7-9. DELATTRE A.-L., « Nécropole punique de la colline Saint-Louis », extrait des Missions Catholiques, Lyon, 1896.

DE MIRO E. et FIORENTTNI G., « Leptis Magna. La necropoli greco-punica sotto il teatro », Quademi di Archeol. délia Libia, 9, 1977, p. 5-75.

FANTAR M.-H., « Nouvelles tombes puniques découvertes sur les flancs de la colline de Bordj Jedid à Carthage », Oriens Antiquus, 10, 1971, 4, p. 313-320.

GILL D.-W.-J., « Attic Black-Glazed Pottery », dans Ph. M. Kenrick, éd., Excavations at Sabratha 1948-1951 (Journal of Roman Studies Monograph n²), Londres, 1986, p. 275- 296.

JODIN A., Les établissements du roi Juba II aux iïes Purpuraires (Mogador), Tanger, 1967 ; Volubilis regia Iubae, Publications du Centre Pierre Paris, 14, Paris, 1987.

KENRICK Ph. M., Excavations at Sidi Khrebish, Benghazi (Bérénice), III, 1, The Fine Pottery (Suppl. to Libya Antiqua, V, III, 1), Tripoli, 1985.

LANCEL S., « Tipasitana III : la nécropole préromaine occidentale de Tipasa. Rapport préliminaire (campagnes de 1966 et 1967) », Bull. d'Arch. Algérienne, 3, 1968, p. 85-166.

MOREL J.-P., «Les niveaux préromains », dans J.-P. CALLU, J.-P. MOREL, R. REBUFFAT et G. HALLIER, Thamusida, fouilles du Service des antiquités du Maroc, Paris, 1965, p. 61-111 ; «Céramiques d'Hippone », Bull. d'Arch. Algérienne, 1, 1962-1965 (1967), p. 107-139 ; «Céramique à vernis noir du Maroc », Ant. Afr., 2, 1968, p. 55-76; " Les céramiques à vernis noir et à figures rouges d'Afrique avant la deuxième guerre punique et le problème des exportations de Grande-Grèce ", Ant. Afr., 15, 1980, p. 29-75 ; Céramique campanienne : les formes, 2 vol., Rome, 1981 ; « La céramique à vernis noir de Carthage-Byrsa : nouvelles données et éléments de comparaison ", Actes du Colloque sur la céramique antique de Carthage (Carthage, 1980), Dossier CEDAC 1, Carthage, 1982, p. 43-76 ; « Les importations de céramiques grecques et italiennes dans le monde punique ( $\mathrm{V}^{\mathrm{e}}-\mathrm{I}^{\mathrm{er}}$ siècles) : révision du matériel et nouveaux documents ", Atti del I Congresso intem. di Studifenici e punici 
(Roma, 1979), vol. III, Rome, 1983, p. 731-740 ; « La céramique à vernis noir de Carthage, sa diffusion, son influence », Cahiers des Études Anciennes, XVIII, Carthage VIII, Actes du Congrès (troisième partie), Trois-Rivières, 1986, p. 25-68 ; « Nouvelles données sur le commerce de Carthage punique entre le VII ${ }^{\mathrm{e}}$ et le $\mathrm{II}^{\mathrm{e}}$ siècle av. J.-C. », Histoire et archéologie de l'Afrique du Nord, Actes du IV Colloque international (Strasbourg, 1988), t. I, Carthage et son territoire dans l'antiquité, Paris, 1990, p. 67-100; « Aperçu sur la chronologie des céramiques à vernis noir aux $\mathrm{II}^{\mathrm{e}}$ et $\mathrm{I}^{\mathrm{er}}$ siècles avant J.-C. », dans A. DUVAL, J.P. MOREL et Y. ROMAN, éd., Gaule interne et Gaule méditerranéenne aux II et I siècles avant J.-C. ; confrontations chronologiques (Actes de la Table ronde de Valbonne, 1986), Revue Arch. de Narbonnaise, suppl. 21, Paris, 1990, p. 55-71; « L'apport des fouilles de Carthage à la chronologie des céramiques hellénistiques ", Devtera Epistimoriki Synantisiya tin ellenistiki Keramiki. Khronologika provlimata tis ellenistikis Keramikis (Rhodes, 1989), Praktika, Athènes, 1990, p. 17-30 ; « La céramique à vernis noir du Maroc : une révision ", Actes du Colloque international «Lixus : bilan et perspectives » (Laroche, 1989), à paraître.

PONSICH M., « Une tombe préromaine des environs de Lixus », Bull. d'Arch. Marocaine, V, 1964, p. 339-342; « Alfarerias de época fenicia y púnico-mauritana en Kuass (Arcila, Marruecos) », Papeles del Laboratorio de Arqueologia de Valencia, 4, Valence, 1968 ; « Note préliminaire sur l'industrie de la céramique préromaine en Tingitane (Kouass, région d'Arcila) », Karthago, 15, 1969, p. 75-97 ; « Les céramiques d'imitation : la campanienne de Kouass, région d'Arcila, Maroc », Archivo Español de Arqueologia, 42, 1969, p. 56-80.

TARRADELL M., Marruecos púnico, Tétouan, 1960.

VILLARD F., « Vases attiques du v viècle av. J.-C. à Gouraya », Libyca, Arch.-Epigr., 7, 1959, 1, p. 7-13 ; «Céramique grecque du Maroc », Bull. d’Arch. Marocaine, IV, 1960, p. 1-26.

\section{INDEX}

Mots-clés : Antiquité, Commerce, Technologie 